La Revista Panamericana de Salud Pública/Pan American Journal of Public Health se complace en publicar cartas de los lectores dirigidas a estimular el diálogo sobre los diversos aspectos de la salud pública en las Américas, así como a esclarecer, discutir o comentar de manera constructiva las ideas expuestas en la revista. Las cartas deben estar firmadas por el autor y especificar su afiliación profesional y dirección postal. Cuando se trate de comentarios sobre un artículo que requieran contestación del autor, se procurará conseguir esa respuesta con el fin de publicar ambas cartas. La Redacción se reserva el derecho de editar las cartas recibidas y resumirlas para mayor claridad.

The Revista Panamericana de Salud Pública/Pan American Journal of Public Health publishes letters from readers for the purpose of stimulating dialogue on various aspects of public health in the Americas and of constructively clarifying, discussing, and critiquing the ideas expressed throughout its pages. Letters should be signed by the author and include his or her professional affiliation and mailing address. If a commentary on a given article requires a reply from the author, an effort will be made to obtain the reply and to publish both letters. The editorial team reserves the right to edit all letters received and to condense them so as to improve their clarity.

\section{FACTOR ESTIMULANTE DE COLONIAS DE GRANULOCITOS EN EL TRATAMIENTO AMBULATORIO DE LA NEUTROPENIA POSTERIOR A LA QUIMIOTERAPIA}

El tratamiento convencional del cáncer contempla la cirugía, la quimioterapia y la radioterapia. Avances recientes en el campo de la biología molecular han permitido desarrollar nuevas opciones terapéuticas basadas en el empleo de proteínas recombinantes, péptidos sintéticos y anticuerpos monoclonales, así como factores inhibidores de la transducción, agentes de diferenciación celular, compuestos antiangiogénicos y protectores celulares, entre otros (1).

Los fármacos antineoplásicos utilizados tradicionalmente inducen mecanismos de apoptosis o de muerte de las células mediante la inhibición de su crecimiento o por los daños que provocan en su ADN. Sin embargo, esta acción no está dirigida de manera específica a las células tumorales y puede tener un efecto tóxico sobre los tejidos sanos, lo que limita la dosis a administrar. La neutropenia es uno de los efectos adversos de la quimioterapia que con mayor frecuencia obliga a aplazar su administración o a reducir la dosis, lo que repercute desfavorablemente en la evolución de los enfermos (2).

El factor estimulante de colonias de granulocitos (FEC-G) es un factor de crecimiento hematopoyético - producido por el organismo en cantidades muy pe- queñas- que regula la formación de los neutrófilos. La producción de FEC-G recombinante ha permitido disponer de las cantidades necesarias para tratar a los pacientes con neutropenia, ya sea esta provocada por la quimioterapia o la radioterapia, o por otros trastornos que afectan a los neutrófilos, como la anemia aplástica, la neutropenia cíclica y la agranulocitosis congénita (3).

Desde 1994, la Sociedad Estadounidense de Oncología Clínica (ASCO) recomienda en sus guías el empleo del FEC-G en el tratamiento de la neutropenia provocada por medicamentos citotóxicos o radiaciones (4), lo que ha permitido que la mayoría de los pacientes reciban el tratamiento de quimioterapia o radioterapia en el tiempo establecido, mejore la atención de los pacientes con neutropenia y disminuya el número de ingresos hospitalarios y el uso de antimicrobianos (5).

Desde hace tres años en el Hospital Provincial Universitario Dr. Gustavo Aldereguía Lima, de la provincia de Cienfuegos, Cuba, se utiliza el ior ${ }^{\circledR}$-LeukoCIM, un FEC-G recombinante producido por el Centro de Inmunología Molecular de Ciudad de La Habana, Cuba.

Este medicamento se administra en forma ambulatoria bajo el control del médico y la enfermera de la familia, por lo que es importante que el personal sanitario de atención primaria conozca las reacciones adversas más frecuentes provocadas por este fármaco, entre ellos dolor óseo, fiebre, hiperuricemia, leucocito- 
sis, neutrofilia, aumento de la enzima lactato deshidrogenasa, trombocitopenia, cefalea, náuseas e hipotensión (6), similares a los informados en la literatura para otros FEC-G (7). En los pacientes tratados en Cienfuegos, estas reacciones se presentaron en menos de $10 \%$ de los casos tratados por neutropenia, con excepción del dolor óseo $(11,1 \%)$ y la fiebre $(11,1 \%)$. En la mayoría de los casos, la intensidad de las reacciones se evaluó como ligera $(76,3 \%)$ y se estableció una posible relación de causalidad $(60,8 \%)$ con la condición clínica de los enfermos $(8,9)$.

Leslie Pérez Ruiz Departamento de Ensayos Clínicos, Centro de Inmunología Molecular Ciudad de La Habana, Cuba Correo electrónico: leslie@cim.sld.cu

Julio D. Fernández Águila Hospital Provincial Universitario Dr. Gustavo Aldereguía Lima Cienfuegos, Cuba

Ana M. Ramos Cedeño Facultad de Ciencias Médicas Dr. Raúl Dorticós Torrado Cienfuegos, Cuba

\section{REFERENCIAS}

1. Katzung BG. Farmacología básica y clínica. En: Chu E, Sartorelli AC. Quimioterapia del cáncer. 9. ${ }^{a}$ ed. México, D.F.: Manual Moderno; 2005. Pp. 885-915.
2. Aapro MS, Cameron DA, Pettengell R, Bohlius J, Crawford J, Ellis M, et al. EORTC guidelines for the use of granulocyte-colony stimulating factor to reduce the incidence of chemotherapyinduced febrile neutropenia in adults patients with lymphomas and solid tumours. Eur J Cancer. 2006;42(15):2433-53.

3. Welte K, Platzer E, Lu L, Gabrilove JL, Levi E. Purification and biochemical characterization of human pluripotent hematopoietic colony stimulating factor. Proc Natl Acad Sci U S. 1985;82: 1526-30.

4. Smith TJ, Khatcheressian J, Lyman GH, Ozer H, Armitage JO, Balducci L, et al. 2006 update of recommendations for the use of white blood cell growth factors: an evidence-based clinical practice guideline. J Clin Oncol. 2006;24:3187-205.

5. Scott S, Chrischilles E, Link B, Delgado D, Fridman M, Stolshek B. Days of prophylactic filgrastim use to reduce febrile neutropenia in patients with non-Hodgkin's lymphoma treated with chemotherapy. J Manag Care Pharm. 2003;9(2 Supl2):15-21.

6. Wilford M, Fernández JD, Mesa R, Muñio J, Figueredo I, Luna $\mathrm{C}$, et al. Evaluación clínica y seguridad del ior GSCF en pacientes con neutropenia grado III-IV por quimio y/o radioterapia. Estudio de extensión. Memorias del V Congreso Nacional y VII Jornada Latinoamericana de Hematología, Inmunología y Medicina Transfusional. 2005 mayo 16-20; Ciudad de La Habana, Cuba. Ciudad de La Habana: Palacio de Convenciones; 2005.

7. Stull D, Bilmes R, Kim H, Fichtl R. Comparison of sargramostim and filgrastim in the treatment of chemotherapy-induced neutropenia. Am J Health Syst Pharm. 2005;62:83-7.

8. Pérez Ruiz L, Ramos AM, Fernández JD, Bobillo H, Fraga O, Guerra $\mathrm{T}$, et al. Intensive pharmacovigilance of grow colony stimulating factor (ior ${ }^{\circledR}$ LeukoCIM) in patients with cancer in Cienfuegos, Cuba. 15th World Congress of Pharmacology. 2006 July 2-7; Beijing, China. Acta Pharm Sin. 2006;27(9):1272-6.

9. Fernández JD, Pérez Ruiz L, Ramos AM, Guerra T, Cabrera M, Prieto J, et al. Impacto del factor estimulante de colonias de granulocitos (ior ${ }^{\circledR}$-Leukocim) en la profilaxis y tratamiento de la neutropenia postquimioterapia en pacientes oncohematológicos de la Provincia Cienfuegos. Rev Cubana Farm. 2007;41 (Supl2):267. 